\title{
Non-finiteness results for Nil-groups
}

\author{
JOACHIM GRUNEWALD
}

\begin{abstract}
Generalizing an idea of Farrell we prove that for a ring $\Lambda$ and a ring automorphism $\alpha$ of finite order the groups $\operatorname{Nil}_{0}(\Lambda ; \alpha)$ and all of its $p$-primary subgroups are either trivial or not finitely generated as an abelian group. We also prove that if $\beta$ and $\gamma$ are ring automorphisms such that $\beta \circ \gamma$ is of finite order then $\operatorname{Nil}_{0}\left(\Lambda ; \Lambda_{\beta}, \Lambda_{\gamma}\right)$ and all of its $p$-primary subgroups are either trivial or not finitely generated as an abelian group. These Nil-groups include the Nil-groups appearing in the decomposition of $K_{i}$ of virtually cyclic groups for $i \leq 1$.
\end{abstract}

18F25; 19B28, 19D35

\section{Introduction}

Let $\Lambda$ be a unital ring and $\alpha$ a ring automorphism. Farrell defined in his $\mathrm{PhD}$ thesis [2] twisted Nil-groups, $\operatorname{Nil}_{i}(\Lambda ; \alpha)$ for $i \in \mathbb{N}$. We denote the twisted polynomial ring by $\Lambda_{\alpha}[t]$. The Nil-group $\operatorname{Nil}_{i}(\Lambda ; \alpha)$ is the kernel of the map $\epsilon: K_{i+1}\left(\Lambda_{\alpha}[t]\right) \rightarrow K_{i+1}(\Lambda)$ which is induced by the augmentation map. Farrell-Hsiang [4] and Grayson [6] generalized the fundamental lemma of algebraic $K$-theory to twisted Laurent polynomial rings. They proved the exactness of the following sequence, relating the $K$-theory of the twisted Laurent polynomial ring $\Lambda_{\alpha}\left[t, t^{-1}\right]$ to the $K$-theory of $\Lambda$ :

$$
\begin{aligned}
\cdots \longrightarrow & K_{i+1}(\Lambda) \stackrel{1-\alpha_{*}}{\longrightarrow} K_{i+1}(\Lambda) \longrightarrow \\
& K_{i+1}\left(\Lambda_{\alpha}\left[t, t^{-1}\right]\right) /\left(\operatorname{Nil}_{i}(\Lambda, \alpha) \oplus \operatorname{Nil}_{i}\left(\Lambda, \alpha^{-1}\right)\right) \longrightarrow K_{i}(\Lambda) \longrightarrow \cdots .
\end{aligned}
$$

Let $A, B$ and $C$ be rings and let $\alpha: C \rightarrow A$ and $\beta: C \rightarrow B$ be inclusions which are pure and free (for a definition of pure and free see Waldhausen [10]). Let $R$ be the push-out of the diagram

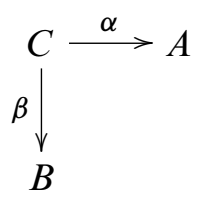

in the category of rings. Waldhausen proved that there is a Mayer-Vietoris sequence for algebraic $K$-theory, which is exact up to Nil-groups $\operatorname{Nil}_{i}\left(C ; A^{\prime}, B^{\prime}\right)$, where $A^{\prime}$ is 
defined to be the $C$-bimodule such that $A=\alpha(C) \oplus A^{\prime}$ and $B^{\prime}$ is defined similarly $[10 ; 11]$. More precisely he proved that the following sequence is exact:

$$
\begin{aligned}
& \cdots \longrightarrow K_{i+1}(C) \longrightarrow K_{i+1}(A) \oplus K_{i+1}(B) \longrightarrow \\
& K_{i+1}(R) / \operatorname{Nil}_{i}\left(C ; A^{\prime}, B^{\prime}\right) \longrightarrow K_{i}(C) \longrightarrow \cdots .
\end{aligned}
$$

In the article at hand we use an idea which goes back to Farrell [3] to prove that Nil-groups and its $p$-primary subgroups have the mysterious property of being either trivial or not finitely generated as an abelian group. For a ring automorphism $\alpha: \Lambda \rightarrow \Lambda$ we denote the $\Lambda$-bimodule $\Lambda$ with $\Lambda$-action from the left via the identity and from the right via $\alpha$ by $\Lambda_{\alpha}$. For an abelian group $G$ and a prime $p$, define

$$
G_{p}=\left\{x \in G: p^{n} x=0 \text { for some } n \geq 0\right\} .
$$

$G_{p}$ is called the $p$-primary subgroup of $G$.

Theorem 1.1 Let $\Lambda$ be a ring, $p$ a prime and $\alpha$ a ring automorphism of finite order. The groups $\operatorname{Nil}_{0}(\Lambda ; \alpha)$ and $\operatorname{Nil}_{0}(\Lambda ; \alpha)_{p}$ are either trivial or not finitely generated as an abelian group. If $\beta$ and $\gamma$ are ring automorphisms such that $\beta \circ \gamma$ is of finite order then $\operatorname{Nil}_{0}\left(\Lambda ; \Lambda_{\beta}, \Lambda_{\gamma}\right)$ and $\operatorname{Nil}_{0}\left(\Lambda ; \Lambda_{\beta}, \Lambda_{\gamma}\right)_{p}$ are either trivial or not finitely generated as an abelian group.

The non-finiteness of $\operatorname{Nil}_{0}(\Lambda ; \alpha)$ for $\alpha=$ id was already known [3] and the nonfiniteness of $\operatorname{Nil}_{0}(\mathbb{Z} G ; \alpha)$ for a finite group $G$ was independently proven by Ramos [9].

For topology the $K$-theory of group rings is of special importance and the Farrell-Jones conjecture, which is known to be true for a large class of groups, predicts that the building blocks of the $K$-theory of a group ring is the $K$-theory of virtually cyclic groups. There are two types of infinite virtually cyclic groups:

(i) the semidirect product $G \rtimes \mathbb{Z}$ of a finite group $G$ and the infinite cyclic group;

(ii) the amalgamated product $G_{1} *_{H} G_{2}$ of two finite groups $G_{1}$ and $G_{2}$ over a subgroup $H$ such that $\left[G_{1}: H\right]=2=\left[G_{2}: H\right]$;

If one decomposes the $K$-theory of infinite virtually cyclic groups the Nil-groups of finite groups appear. Since for a finite group all automorphisms are of finite order we obtain the following corollary about Nil-groups of finite groups.

Corollary 1.2 Let $R$ be a ring, $G$ a finite group, $p$ a prime and $\alpha$ and $\beta$ group automorphisms. The groups $\operatorname{Nil}_{i}(R G ; \alpha), \operatorname{Nil}_{i}(R G ; \alpha)_{p}, \operatorname{Nil}_{i}\left(R G ; R G_{\alpha}, R G_{\beta}\right)$ and $\mathrm{Nil}_{i}\left(R G ; R G_{\alpha}, R G_{\beta}\right)_{p}$ are either trivial or not finitely generated as an abelian group for $i \leq 0$. 
For $R=\mathbb{Z}$ the considered Nil-groups are known to vanish for $i \leq-2$ (see Farrell and Jones [5]) and are known to be $n$-torsion for an arbitrary group of finite order $n$ (see Kuku and Tang [8]).

\section{Non-finiteness results for Nil-groups}

In the following $\Lambda$ will always be a unital ring and $\alpha$ a ring automorphism of finite order $n$, that is, $\alpha^{n}=\mathrm{id}$.

For $m \in \mathbb{N}$ we have canonical inclusion maps

$$
\sigma_{m}: \Lambda_{\alpha^{n m+1}}\left[t^{n m+1}\right] \rightarrow \Lambda_{\alpha}[t] .
$$

Those maps induce transfer and induction maps

$$
\begin{aligned}
\sigma_{*}^{m}: K_{1}\left(\Lambda_{\alpha^{n m+1}}\left[t^{n m+1}\right]\right) & \rightarrow K_{1}\left(\Lambda_{\alpha}[t]\right) \\
\sigma_{m}^{*}: K_{1}\left(\Lambda_{\alpha}[t]\right) & \rightarrow K_{1}\left(\Lambda_{\alpha^{n m+1}}\left[t^{n m+1}\right]\right) .
\end{aligned}
$$

Since $\operatorname{Nil}_{0}(\Lambda ; \alpha)=\operatorname{Nil}_{0}\left(\Lambda ; \alpha^{n m+1}\right)$ we have an embedding

$$
\iota^{\prime}: \operatorname{Nil}_{0}(\Lambda ; \alpha) \hookrightarrow K_{1}\left(\Lambda_{\alpha^{n m+1}}\left[t^{n m+1}\right]\right) .
$$

The proof of the non-finiteness result is based on the following diagram:

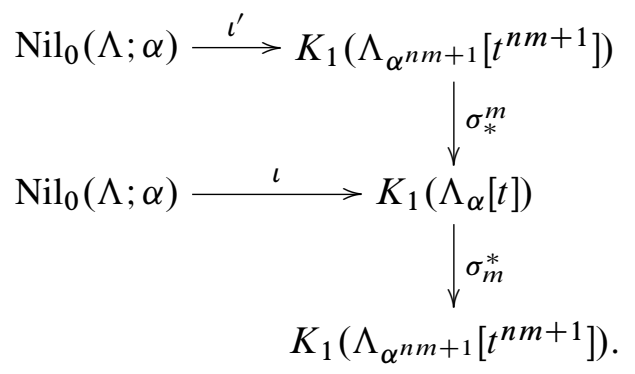

The idea is to choose, for a finitely generated Nil-group, $m$ such that $\sigma_{m}^{*} \sigma_{*}^{m} \iota^{\prime}$ is a monomorphism (Lemma 2.1) and trivial (Proposition 2.3). Thus every finitely generated Nil-group is trivial.

Lemma 2.1 Let $G$ be a finitely generated subgroup of $K_{1}\left(\Lambda_{\alpha^{n m+1}}\left[t^{n m+1}\right]\right)$. For every $K \in \mathbb{N}$ there is an $m \geq K$ such that $\sigma_{m}^{*} \sigma_{*}^{m}$ is a monomorphism on $G$.

Proof Let $T$ be the exponent of the torsion subgroup of $G$ and let $F$ be the rank of a maximal torsion free subgroup. Choose $\ell \in \mathbb{N}$ such that $\ell \cdot T \geq K$. For $x \in$ 
$K_{1}\left(\Lambda_{\alpha^{n m+1}}\left[t^{n m+1}\right]\right)$ we have

$$
\sigma_{\ell \cdot T}^{*} \sigma_{*}^{\ell \cdot T}(x)=\sum_{i=0}^{n \cdot \ell \cdot T} \alpha_{*}^{i}(x)=x+\ell \cdot T \sum_{i=1}^{n} \alpha_{*}^{i}(x)
$$

where $\alpha_{*}: K_{1}\left(\Lambda_{\alpha^{n m+1}}\left[t^{n m+1}\right]\right) \rightarrow K_{1}\left(\Lambda_{\alpha^{n m+1}}\left[t^{n m+1}\right]\right)$ is the map which is induced by the ring automorphism on $\Lambda_{\alpha^{n m+1}}\left[t^{n m+1}\right]$ which sends an element $\sum r_{i} t^{i}$ to the element $\sum \alpha\left(r_{i}\right) t^{i}$. The automorphism $\alpha_{*}$ restricts to an automorphism of $A:=$ $\cup_{i=1}^{n} \alpha^{i}\left(\mathbb{Z}^{F}\right)$ where $\mathbb{Z}^{F}$ is a maximal torsion free subgroup of $G$. The map $\left.\alpha_{*}\right|_{A}$ is conjugate to a diagonal matrix, that is,

$$
g \alpha_{*} \mid{ }_{A} g^{-1}=\left(\begin{array}{ccc}
\zeta_{1} & & \\
& \ddots & \\
& & \zeta_{r}
\end{array}\right)
$$

where $g \in \mathrm{GL}_{r}(\mathbb{C})$ and $\zeta_{1}, \ldots, \zeta_{r} \in \mathbb{C}$ are $n$th roots of unity. We can find $k \in \mathbb{N}$ such that $\sum_{i=1}^{k \cdot \ell \cdot T} \zeta_{j}^{i} \neq-1$ for all $j \in\{1, \ldots, r\}$. One verifies easily that $\sigma_{k \cdot \ell \cdot T}^{*} \sigma_{*}^{k \cdot \ell \cdot T}(x)$ is a monomorphism.

Lemma 2.2 The image of $\iota^{\prime}$ is mapped into the image of $\iota$ by every $\sigma_{*}^{m}$.

Proof The result follows since the diagram

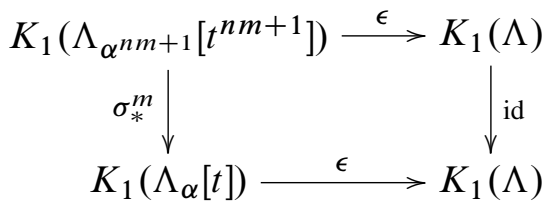

commutes. We denote the maps which are induced by the augmentation map by $\epsilon$.

Proposition 2.3 For every $x \in \operatorname{Nil}_{0}(\Lambda ; \alpha)$, there exists an integer $K(x)$ such that $\sigma_{m}^{*}(x)=0$ for all integers $m \geq K(x)$.

For the proof of Proposition 2.3 we need the following lemma. We denote by $\operatorname{GL}_{n}(\Lambda)$ the group of invertible $n \times n$ matrices, by $\operatorname{GL}(\Lambda)$ the colimit over $\operatorname{GL}_{n}(\Lambda)$ and by $\mathrm{E}\left(\Lambda_{\alpha}[t]\right)$ the subgroup of $\mathrm{GL}\left(\Lambda_{\alpha}[t]\right)$ generated by all elementary matrices. For a matrix $N$ we denote by $\alpha(N)$ the matrix obtained for $N$ by applying $\alpha$ to each component.

Lemma 2.4 Every matrix $B \in \mathrm{GL}\left(\Lambda_{\alpha}[t]\right)$ can be reduced, modulo GL( $\left.\Lambda\right)$ and $\mathrm{E}\left(\Lambda_{\alpha}[t]\right)$, to a matrix of the from $1+N t$, where

$$
\prod_{j=0}^{M} \alpha^{-j}(N)=0
$$

Algebraic ${ }^{3} \mathcal{G}$ Geometric Topology, Volume 7 (2007) 
for some $M \in \mathbb{N}$.

Proof We have

$$
B=B_{0}+B_{1} t+\ldots+B_{n} t^{n}
$$

with $B_{i} \in \operatorname{Mat}_{m}(\Lambda)$. In $\operatorname{GL}\left(\Lambda_{\alpha}[t]\right)$ we have

$$
B=\left(\begin{array}{cc}
B & 0 \\
0 & \text { id }
\end{array}\right)
$$

Modulo $\mathrm{E}\left(\Lambda_{\alpha}[t]\right)$ we have:

$$
\left(\begin{array}{cc}
B & 0 \\
0 & \text { id }
\end{array}\right)=\left(\begin{array}{cc}
B & B_{n} t^{n} \\
0 & \text { id }
\end{array}\right)=\left(\begin{array}{cc}
B-B_{n} t^{n} & B_{n} t^{n} \\
-t & \text { id }
\end{array}\right) .
$$

This implies by induction that

$$
B=\widetilde{B}_{0}+\widetilde{B}_{1} t .
$$

Since $B \in \mathrm{GL}_{k}\left(\Lambda_{\alpha}[t]\right)$ there exists $B^{-1}$ with

$$
B^{-1}=C_{0}+C_{1} t+\ldots+C_{m} t^{m} .
$$

where $C_{i} \in \operatorname{Mat}_{k}(\Lambda)$. We have

$$
1=B B^{-1}=B_{0} C_{0}+B_{1} t C_{0}+\ldots+B_{1} t C_{m} t^{m} .
$$

Thus $B_{0} C_{0}=1$ and therefore $B=1+N t$ module GL( $\left.\Lambda\right)$. Let $L=L_{0}+L_{1} t+$ $\ldots+L_{m} t^{m}$ be the inverse of $(1+N t)$. We have

$$
\begin{aligned}
1 & =(1+N t)\left(L_{0}+L_{1} t+\ldots+L_{m} t^{m}\right) \\
& =L_{0}+N t L_{0}+L_{1} t+N t L_{1} t+\ldots+L_{m} t^{m}+N t L_{m} t^{m} \\
& =L_{0}+\sum_{i=0}^{m-1}\left(N \alpha^{-1}\left(L_{i}\right)+L_{i+1}\right) t^{i+1}+N \alpha^{-1}\left(L_{m}\right) t^{m+1}
\end{aligned}
$$

This implies the following identities:

$$
\begin{aligned}
& L_{0}=1 \\
& N \alpha^{-1}\left(L_{0}\right)+L_{1}=0 \vdots \\
& N \alpha^{-1}\left(L_{i}\right)+L_{i+1}=0 \vdots \\
& N \alpha^{-1}\left(L_{m-1}\right)+L_{m}=0 \\
& N \alpha^{-1}\left(L_{m}\right)=0 .
\end{aligned}
$$

Algebraic $8 \mathcal{G}$ Geometric Topology, Volume 7 (2007) 
Thus

$$
\prod_{j=0}^{m-1} \alpha^{-j}(N)=0
$$

Proof of Proposition 2.3 By Lemma 2.4 we have

$$
x=1+N t
$$

with

$$
\prod_{i=0}^{M} \alpha^{-i}(N)=0 .
$$

The element $\sigma_{*}^{m}(x)$ is represented by the matrix

$$
\left(\begin{array}{ccccc}
\text { id } & & & & \alpha^{-n m}(N) t^{n m+1} \\
N & \text { id } & & & \\
& \alpha^{-1}(N) & \ddots & & \\
& & \ddots & \text { id } & \\
& & & \alpha^{-n m+1}(N) & \text { id }
\end{array}\right) .
$$

Thus $\sigma_{*}^{m}(x)$ is also represented by the following matrix:

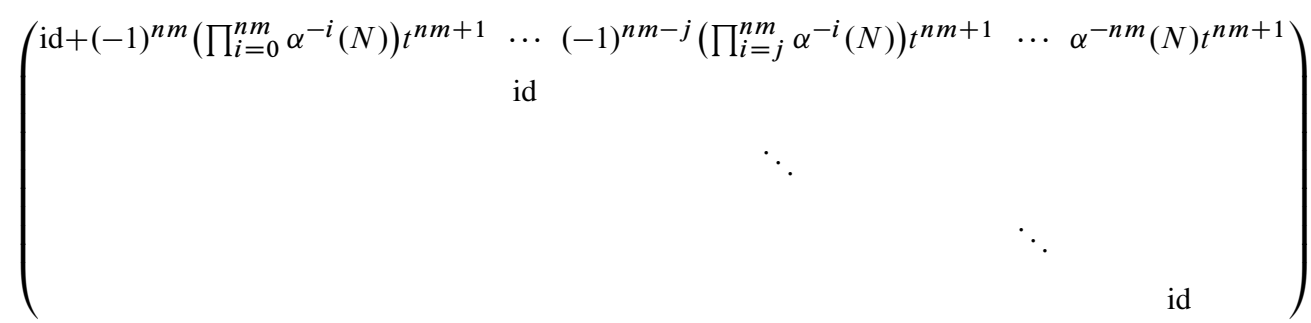

This implies that for $m$ such that $n \cdot m \geq M$ we have $\sigma_{*}^{m}(x)=0$.

Theorem 2.5 Let $\Lambda$ be a ring, $p$ a prime and $\alpha$ a ring automorphism of finite order. The groups $\operatorname{Nil}_{0}(\Lambda ; \alpha)$ and $\operatorname{Nil}_{0}(\Lambda ; \alpha)_{p}$ are either trivial or not finitely generated as an abelian group.

Proof Assume $\operatorname{Nil}_{0}(\Lambda ; \alpha)$ to be a finitely generated abelian group. By Lemma 2.2 and Proposition 2.3 we can find $K$ such that $\sigma_{m}^{*} \sigma_{*}^{m} \iota^{\prime}(x)=0$ for all $x \in \operatorname{Nil}(\Lambda ; \alpha)$ and $m \geq K$. By Lemma 2.1 we can find an $m \geq K$ such that $\sigma_{m}^{*} \sigma_{*}^{m} \iota^{\prime}$ is a monomorphism. Thus $\operatorname{Nil}_{0}(\Lambda ; \alpha)$ is the trivial group. The proof for $\operatorname{Nil}(\Lambda ; \alpha)_{p}$ goes in exactly the same way.

Algebraic ${ }^{3} \mathcal{G}$ Geometric Topology, Volume 7 (2007) 
Corollary 2.6 Let $\Lambda$ be a ring, $p$ be a prime and $\alpha$ and $\beta$ be ring automorphisms such that $\alpha \circ \beta$ is of finite order. The groups $\operatorname{Nil}_{0}\left(\Lambda ; \Lambda_{\alpha}, \Lambda_{\beta}\right)$ and $\operatorname{Nil}_{0}\left(\Lambda ; \Lambda_{\alpha}, \Lambda_{\beta}\right)_{p}$ are either trivial or not finitely generated as an abelian group.

Proof It is a result of Kuku and Tang [8] that $\operatorname{Nil}_{0}\left(\Lambda ; \Lambda_{\alpha}, \Lambda_{\beta}\right)$ can also be described as a Nil-group of type $\operatorname{Nil}_{0}(\Lambda \times \Lambda ; \gamma)$ where $\gamma$ is the ring automorphism defined by

$$
\gamma:(a, b) \mapsto(\beta(b), \alpha(a)) .
$$

Corollary 2.7 Let $R$ be a ring, $G$ a finite group, $p$ a prime and $\alpha$ and $\beta$ group automorphisms. The groups $\operatorname{Nil}_{i}(R G ; \alpha), \operatorname{Nil}_{i}(R G ; \alpha)_{p}, \operatorname{Nil}_{i}\left(R G ; R G_{\alpha}, R G_{\beta}\right)$ and $\mathrm{Nil}_{i}\left(R G ; R G_{\alpha}, R G_{\beta}\right)_{p}$ are either trivial or not finitely generated as an abelian group for $i \leq 0$.

Proof Using the suspension ring construction as explained by Bartels and Lück [1] and by the author [7], one gets that the considered Nil-groups are covered by Theorem 2.5 and Corollary 2.6.

\section{References}

[1] A Bartels, W Lück, Isomorphism conjecture for homotopy $K$-theory and groups acting on trees, J. Pure Appl. Algebra 205 (2006) 660-696 MR2210223

[2] F T Farrell, The obstruction to fibering a manifold over a circle, Indiana Univ. Math. J. 21 (1971/1972) 315-346 MR0290397

[3] F T Farrell, The nonfiniteness of Nil, Proc. Amer. Math. Soc. 65 (1977) 215-216 MR0450328

[4] F T Farrell, W-C Hsiang, A formula for $K_{1} R_{\alpha}[T]$, from: "Applications of Categorical Algebra (Proc. Sympos. Pure Math., Vol. XVII, New York, 1968)”, Amer. Math. Soc., Providence, R.I. (1970) 192-218 MR0260836

[5] F T Farrell, L E Jones, The lower algebraic $K$-theory of virtually infinite cyclic groups, K-Theory 9 (1995) 13-30 MR1340838

[6] D R Grayson, The K-theory of semilinear endomorphisms, J. Algebra 113 (1988) 358-372 MR929766

[7] J Grunewald, The Behavior of Nil-Groups under Localization and the Relative Assembly Map, Preprintreihe SFB 478-Geometrische Strukturen in der Mathematik, Münster, Heft 429 (2006)

[8] A O Kuku, G Tang, Higher K-theory of group-rings of virtually infinite cyclic groups, Math. Ann. 325 (2003) 711-726 MR1974565

[9] R Ramos, Non Finiteness of twisted Nils, preprint (2006) 
[10] F Waldhausen, Algebraic K-theory of generalized free products I, II, Ann. of Math. (2) 108 (1978) 135-204 MR0498807

[11] F Waldhausen, Algebraic $K$-theory of generalized free products III, IV, Ann. of Math. (2) 108 (1978) 205-256 MR0498808

Fachbereich Mathematik und Informatik, Westfälische Wilhelms-Universität Münster Einsteinstrasse 62, D-48149 Münster, Germany

grunewal@math. uni-muenster.de

Received: 6 May 2006 